\title{
Will Love Tear Them Apart? Identifying the Eco-Evolutionary Feedbacks of Sexual Traits
}

João Alpedrinha ${ }^{1}$, Leonor Rodrigues ${ }^{1}$, Sara Magalhães ${ }^{1}$, Jessica Abbott ${ }^{2}$.

1. cE3c: Centre for Ecology, Evolution, and Environmental Changes, Faculty of Sciences, University of Lisbon, Edifício C2, 3 piso, 1749-016 Lisboa, Portugal

2. Biology Department, Section for Evolutionary Ecology, Lund University, Sölvegatan 37, 22362 Lund, Sweden

\section{Declarations}

This project was funded by an ERC grant (COMPCON GA725419) to S Magalhães and by an ERC grant (ERC-StG-2015-678148 (ComplexSex)), to J Abbottt. J Alpedrinha had a Post-Doc grant (SFRH/BPD/109533/2015) funded by FCT (Fundação para a Ciência e Tecnologia). J Abbott also funded by Swedish Research Council (VR-2015-04680).

\section{Abstract}

The study of eco-evolutionary feedbacks is in clear recent expansion. However, most studies concern predator-prey and host-parasite interactions, while the analysis of eco-evolutionary 
feedbacks involving sexual interactions is lagging behind. This is at odds with the potential of these interactions to engage in such processes. Indeed, there is now ample evidence that sexual selection is affected by ecological change. There is also evidence that sexual selection traits evolve rapidly, which may modify the ecological context of species, and thus the selection pressures they will be exposed to. Here, we first set a clear distinction between processes in which ecology drives evolution and those in which the contemporary evolution of populations may change their ecology, depending on which traits act as drivers and objects of change. We then review evidence for these processes and discuss examples of closed eco-evolutionary feedbacks in an attempt to understand how we can tear this loop apart. We suggest that a better understanding of eco-evolutionary feedbacks of sexual selection may help us understand the effects of sexual selection on the rate of adaptation, speciation, and extinction, and thus foster future research in this area.

Keywords: sexual selection, sexual conflicts, contemporary evolution, mate choice, mating systems, mating strategies. 


\section{Introduction}

Traditionally, the field of evolutionary ecology has considered that ecology and evolution operate at separate time-scales. Studies have thus focused on the effect of ecological change on the evolution of populations, assuming that evolutionary changes in ecological time were negligible (Slobodkin 1961, Pelletier et al. 2009, Hendry 2016, Lion 2017). Hothe last decades have witnessed recognition that ecology and evolution can operate at similar time scales, such that evolutionary changes are contemporary with ecological changes, raising the possibility of eco-evolutionary feedbacks (Fuing of timescales is currently so widely accepted that the terms "eco-evolutionary feedbacks" and "eco-evolutionary dynamics" are often used as synonyms of evolutionary ecology (Henfortunate, as it undermines the value of these terms. Here, we define "eco-evolutionary feedbacks" as the contemporary reciprocal effect a) of an ecological factor (be it biotic or abiotic) upon the evolutionary trajectory of a population, and b) of this evolutionary change on an ecological factor. Note that the initial and final factors are not necessarily the same, and that the same eco-evolutionary feedback can affect different traits, individuals or species. This term thus not only encompasses tight evolutionary interactions traditionally defined as co-evolution, but also indirect ecological interactions, such as apparent competition. We further divide eco-evolutionary feedbacks in evo-to-eco and eco-to-evo processes, whenever we refer to a) or b) respectively. "Eco-evolutionary dynamics" occur when the dynamics of genetic change happen on approximately the same time scale as the dynamics of ecological change, and each is a function of the other. Consequently, this term refers to an essentially continuous phenomenon, composed of multiple sequential eco-evolutionary feedbacks. Although eco-evolutionary dynamics are pervasive and an integral part of the 
evolution of populations, characterizing the eco-evolutionary feedback processes of which they are composed is not a simple task.

Sexual selection can be a fertile ground for the occurrence of feedbacks between ecology and evolution. First, it concerns the interaction between two sexes in which one can be part of the ecology of the other, and in which fast evolutionary dynamics have been amply demonstrated, sexual selection is strongly intertwined with the ecological setting of populations. This has been known ever since Darwin defined and laid the foundations of sexual selection research also increasingly evident that the evolution of traits under sexual selection can take place rapidly and potentially modify the ecological context and the selection pressures to which species are exposed to.(see section on "Ecology as a driver of evolution" below).

It is the same entwined nature of the ecological and evolutionary effects of sexual selection that often makes them difficult to disentangle. This problem emerges across different approaches used to study sexual selection, regardless of whether they are field observations, experimental evolution, or phylogenetic analyses. As a consequence, researchers often use different terms and definitions to refer to the same process, putting emphasis on either a particular ecological setting or event, or its evolutionary pressure. This may lead to ambiguities and even different conclusions about the nature of the process being studied. For instance, the same work can conclude that ecology drives the evolution of a trait or the reverse. Thus, identifying ecoevolutionary feedbacks requires a clear distinction between traits that function as the object or the driver of a process. One can easily imagine a scenario where a change in one abiotic factor (e.g., visibility, temperature, light) alters the value of sexually-selected traits, thus modifying the selection pressures in the study population. In this scenario, an ecological factor imposes a selective pressure on a population, where the abiotic factor acts as a driver of change in the sexually-selected trait. The altered sexually selected trait may then in turn act as a driver of change in some other trait. In the first stage, identifying the driver and object is relatively simple, 
but in the second stage, the sexually selected trait switches from object to driver. In general, traits involved in biotic interactions will be harder to classify, since they can be either objects or drivers of ecological or evolutionary change at any given point (Figure 1).

Finally, it is important to distinguish between sequentially-evolved and co-varying traits (Figure 1). For instance, at high density, female survival may evolve a) as a defensive trait due to the evolution of increased male aggressiveness (sequential change), b) because of an increase in density per se (co-variation), or c) due to another factor altogether. Hence, an accurate definition of causation is essential to the experimental design of any eco-evolutionary study, being it observational or manipulative.

In this manuscript we discuss potential eco-evolutionary feedbacks in sexual selection. We first set a clear distinction between processes in which ecology drives evolution and those in which the contemporary evolution of populations may change their ecology, depending on which traits act as drivers and objects of change. We then discuss how to distinguish such processes and use biological examples of sexual selection as case studies. Furthermore, we consider common research methods to establish their advantages and limitations. Our goal here is to establish a systematic (and as unambiguous as possible) approach to the study of eco-evolutionary feedbacks in the context of sexual selection.

\section{Defining the battleground: methodology}

To identify an eco-evolutionary feedback, it is essential to clearly distinguish eco-to-evo and evoto-eco processes. As pointed out before, this is often difficult owing to the entwined nature of 
ecological and evolutionary dynamics. Here, we highlight two complementary approaches equipped to deal with such complexity.

Experimental evolution provides a degree of environmental and population control that is particularly suited to unravel eco-evolutionary feedbacks. Indeed, most experimental evolution studies start by imposing different selective pressures to replicate populations originating from a common stock population. After some generations, individual and population traits are measured in one or several environments. Often, different selective pressures lead to significant differences in trait values. Hence, at their onset, experimental evolution studies are examples of eco-to-evo processes. Commonly enough though, they lead to new ecological dynamics that provide empirical evidence for evo-to-eco processes. In those cases, experimental evolution provides unambiguous evidence of eco-evolutionary feedbacks.

Another means to identify an eco-evolutionary process is to thoroughly compare populations of the same species that are exposed (or not, as a control) to a specific ecological factor which causes evolutionary changes in one or more traits, and then study the consequences of these evolutionary changes. Apart from necessitating a deep knowledge of the populations involved, this approach will often have limitations in determining both causality and a contemporary relationship between the traits involved but may be useful in species where experimental evolution is not an option. In addition, causality may in some cases be determined via further experimental manipulations.

\section{Ecology as a driver of selection}

For an evolving population, ecological factors are environmental traits that impose a selection pressure on the individuals of the population. Such traits can be: 1) abiotic: such as light, temperature, humidity, or patchiness of the habitat; 2) biotic, heterospecific: including 
predators, parasites, prey, or heterospecific competitors; 3) biotic, conspecific: including intraspecific competitors (for food, mates, territory), population density, or sex ratio. Note that abiotic factors may have a biotic origin (for instance, oxygen depletion or reduced visibility in aquatic systems as a consequence of algae blooms).

Many species use environmentally-mediated cues as social signals, to detect and choose potential mating partners and competitors. Such signals can be auditory, chemical or visual in nature. Changes in the environment may modify the effectiveness of such systems directly or indirectly. For instance, a change in water temperature may increase the energetic costs of keeping a territory and decrease the mating success of territorial fishes, even though the transmission efficiency of chemical or visual signals may not be directly affected. Alternatively, as in cichlid fishes of the East African lakes, changes in the environment have been shown to directly affect diversification within the genus Pundamilia. In this genus, female preference for male colour is necessary and sufficient for assortative mating between Pundamilia pundamilia and Pundamilia nyererei, two closely related species with gene flow comparison between two populations of $P$. pundamilia showed that male coloration preference was significantly weaker in females originating from turbid water populations than for females from clear water populations rthermore, a mesocosm experiment with $P$. nyererei showed that male mating success could be predicted by male coloration gether, these findings suggest that variation in water transparency affects sexual selection in these fish. However, by itself this is not an eco-toevo process. Evolution is defined by changes in the frequency of genetically-determined phenotypes over generations and to clearly demonstrate an eco-to-evo process, one needs to either (a) map the genetic basis of the target phenotype or (b) measure trait values while 
removing any environmental effect inducing plastic changes in the trait. The latter is generally done via reciprocal transfers or common garden experiments

Unlike abiotic factors, biotic factors can be both ecological drivers and objects of selection (Figure 1). It may thus prove difficult to tear these two roles apart. For instance, male harassment is a trait under sexual selection due to male-to-male competition. At the same time, from the point of view of females, male harassment is an ecological trait that imposes a fitness cost on females, resulting in a conflict between the sexes. Under stronger sexual conflict, females are expected to evolve traits that increase their resistance or decrease their exposure to male harassment. These phenotypic female traits, in turn, are part of the male's ecology as well, and they can drive male evolution, and so on. Hence, one must be very precise in determining the context in which a trait is under selection (i.e. the object of selection) and the context in which it acts as a selective pressure (i.e. the driver of selection).

A recent study in Drosophila melanogaster provides a good example for the identification of the factors involved in a feedback loop this study, eight replicated populations evolving in different environments (with cadmium or ethanol) showed divergence in sex-related traits. Although no evidence was found for any variation in male reproductive traits (sperm offense or defense), females adapted to one environment (ethanol) tended to produce a higher proportion of offspring sired by their first mate as compared to those adapted to the other environment (cadmium). This suggests that trait divergence occurred in response to a change in diet, successfully excluding male evolution as the driver of selection in females.

Frequently, though, eco-to-evo processes are merely hinted at. Still, acknowledging such hints can be a necessary first step to fully identify such processes. Lesna \& Sabelis' k on the predatory mite Hypoaspis aculeifer can be considered one of the first studies of an eco-to-evo process involving sexual selection. In this study, genetically-based diversity in prey preference was found 
in a field population of soil predatory mites, in which some females preferred to prey upon the bulb mite Rhizoglyphus robini ( $\mathrm{R}$ line), while others preferred to prey upon the copra mites Tyrophagus putrescentiae ( $T$ line). They found that females placed in a novel diet mate disassortatively, producing more successful offspring. However, on their native diet, mites mated assortatively. In this way, good genes were always ensured for the offspring. In this system, thus, an eco-to-evo process could result from the fitness advantage that emerges from the mating preference.

\section{Evolution as driver of ecological change}

One of the main goals of eco-evolutionary feedbacks is to determine how contemporary evolutionary responses can influence ecological traits, at the population, community and ecosystem levels reveal an evo-to-eco process, the evolution of a trait needs to be studied and complemented by an analysis of its ecological consequences. These do not necessarily need to be related to sexual selection and can even be abiotic in nature. Nevertheless, determining a direct causal relationship between the evolution of a trait and its ecological consequence in a controlled setting is key. To accomplish this, first it is necessary to detect or manipulate a contemporary evolutionary response. The existence of an evo-to-eco process thus necessarily implies a previous switch in ecological pressure (which initially caused the evolutionary change), indicating that this process is inevitably part of an eco-evolutionary feedback. Studies including these feedbacks, whether inadvertently or not, are thus presumably more frequent than has originally been thought. However, explicitly demonstrating the existence of an eco-evolutionary feedback can be challenging, even under controlled experimental conditions (see Discussion).

Several studies have focused on the role of sexual selection on ecological adaptation, revealing an evo-to-eco process. For example, using an experimental evolution approach, Plesnar-Bielak 
et al. owed that populations of bulb mites (Rhizoglyphus robini) evolving under enforced monogamy were less resilient when facing a thermal environmental challenge than polygamous lines. In this study, a factorial design was used to establish populations evolving under strong (females are allowed to mate with several males; polygamy) versus weak (females mated only once; monogamy) sexual selection and under standard versus challenging (high) temperature conditions. They found that within 17 generations, all populations evolving under weak sexual selection and subject to high-temperature conditions had gone extinct. In contrast, all polygamous populations survived. Thus, the decline in fertility in the monogamous regime was shown to be associated with decreased purging of deleterious alleles via sexual selection and/or reduced adaptive ability due to the production of fewer allelic combinations from the available standing genetic variation. Much in the same way, Lumley et al. Iso using experimental evolution, investigated how sexual selection influenced the ability of populations to withstand inbreeding in the flour beetle Tribolium castaneum. They manipulated sexual selection by creating the following regimes: male-biased (strong sexual selection), female-biased (weak sexual selection), and polygamous (strong sexual selection) or monogamous (weak sexual selection) populations. Populations were maintained under experimental evolution regimes for 45 generations, at the end of which they were subjected to inbreeding (sib $x$ sib mating) for up to 20 generations. While lineages under weak sexual selection showed a rapid decline in fitness, lineages under strong selection were significantly more resilient to the mutational load. Importantly, the authors confirmed that during the first 45 generations, effective population size and heterozygosity were maintained as identical between treatments. Both studies strongly suggest that sexual selection alleviates the effects of inbreeding, thus allowing these populations to avoid extinction.

Another good example of a study exploring an evo-to-eco process is one by Heathcote and colleagues ho explored the effect of sexual selection on population co-existence. Here, sexual 
selection acting on traits involved in male-to-male competition was shown to promote mate choice and assortative mating between diverging populations. This was observed in the contact zone between two lineages of the European lizard Podarcis muralis, where males from the Italian lineage are larger in size and exhibit greater exaggeration of intra-sexually selected secondary characters ese males are competitively dominant over the Atlantic lineage, and hybridization occurs mainly between males from the dominant population and females from the less competitive population. In this case, sexual selection between two populations results in a change in behaviour, potentially leading to asymmetric introgression between populations.

\section{Examples of eco-evolutionary feedbacks of sexual selection}

Demonstrating a feedback loop is not trivial, since it requires establishing the appropriate ecological driver, measuring selection on various relevant traits in order to identify potential objects of selection, documenting an evolutionary response to selection in some or all of these traits, while disentangling causal and correlated responses within the feedback loop (tearing the loop apart). In addition, feedbacks may occur via the action of more than one driver, for example if resource availability influences mating system, and subsequently the nature of intraspecific competition. Such "mixed" feedbacks will probably be common in nature but somewhat more complex to analyze.

As a hypothetical example, consider a given experimental evolution design with two regimes: in one of them, females are allowed to mate with several males (polygamy, high sexual selection) while in the other, females mate with a single male (monogamy, low sexual selection). After some generations, males from the multiple mating regime show increased mating rate. Females from the single mating treatment also have higher mortality when crossed with males from the 
multiple mating treatment as compared to females from the multiple mating treatment that mated with the same males. Laying out all the events, we have:

1) Two ecological regimes that impose different selective pressures.

2) The multiple mating regime selects for more aggressive males due to stronger intrasex competition.

3) The evolutionary response of females, with females from the multiple mating regime showing higher resistance to male harm than females from the single regime.

From an initial change in an ecological factor (1), several causality cascades are possible with this simple example (Figure 2). First, both 2 and 3 may evolve as a response to the selection pressure described in 1 . In this case, we cannot consider this as a feedback loop, but just evidences of 2 traits jointly evolving in response to a common selection pressure (i.e. an ecological factor is the driver of selection and both aggressiveness in males and resistance in females are the objects of selection). The other possibility is that 1 is a selection pressure for just one of those traits, either 2 or 3 , and the other responds to this selected trait. For example, if trait 2 is the object of the initial selection pressure, but then in turn becomes the ecological driver of evolutionary change in trait 3 (or vice versa). This is a good example of an ecoevolutionary feedback. The way to disentangle these two options is not straightforward. Indeed, one would need to show that the responding trait (3) would not evolve in populations exposed to the same ecological conditions (1), but in which the selected trait (2) did not evolve. One would not expect females to evolve resistance to male harassment in an environment where females mate multiply, but in which males do not harass females.

The seminal work by Bill Rice has managed to disentangle coevolution between males and females and expose the feedback Rice's Drosophila populations, coevolution of the two sexes was prevented using genetic manipulation, allowing males to evolve but preventing females 
from doing so. Females were thereby transformed into an ecological resource for males, but their role as an evolutionary player was abolished. In the face of non-evolving females, males evolved an increased re-mating rate and increased seminal fluid toxicity, which reduces female survival. Thus, blocking the females' response exposed the evolution of male harassment/harm. Furthermore, because under male and female coevolution these negative effects of males upon females are not detected, these results suggest that females respond to the evolution of male harassment and harm by becoming resistant. Yet, with this study it is not possible to determine the driver of male harassment: males can have evolved in response to a change in female's resistance, due to increase male-male competition or a combination of both.

Below, we discuss three empirical examples of eco-evolutionary feedbacks of sexual selection,each for a given type of driver of change (abiotic, heterospecific, or conspecific).

\section{Abiotic factors}

In the introduction, we discussed an example in Pundamilia where water turbidity influenced sexual selection on male colour. However, what was missing from this example, in order to establish the existence of a feedback loop, was the demonstration that the resulting phenotypic changes were genetic, not plastic. In contrast, work by Seehausen et al. evealed the full feedback loop in this genus. Indeed, they found that deeper water selects for better detection of blue light, presumably via more efficient foraging. This change in female sensory perception then selects for altered male nuptial colouration (more blue in deep water, more red in shallow water), in order to increase conspicuousness during courtship. Female preference for males of a given colour leads to speciation along a depth gradient, and opsin sequence and microsatellite data confirm that these changes have a genetic basis. In this case, we have an example of an 
eco-evolutionary feedback mediated by an abiotic factor: eco (light environment at different depths) to evo (altered opsin sensititivity) to eco (speciation and habitat specialization).

Heterospecifics

Magurran \& Seghers died the reproductive behaviour of the guppy Poecilia reticulata in the wild. Several traits, such as the number of mating attempts in males and the frequency of mating avoidance by females, were compared across seven populations differing in risk of predation. In locations with high predation risk, in which sex-ratio tends to be more evenly balanced (lowpredation sites tend to be female-biased; males suffered more harassment from males and evaded more mating attempts. Thus, this shows that sexual conflicts evolved in the presence of predators, probably because of changes in the guppies' sex-ratio and a decrease in males' energetic needs owing to their smaller size. Female evasion has a cost: it leads to decreased foraging by females, resulting in a poorer diet is study is thus exposing an effect of evolution of a sexual trait, or traits, on the species ecology. It is therefore an example of an eco-evolutionary feedback mediated by heterospecific interactions: eco (predation risk) to evo (sexual conflicts) to eco (change in diet).

Conspecifics

Martin \& Hosken ipulated population density in the dung fly Sepsis cynipsea for 42 generations, after which they measured a number of reproductive traits. By comparing the same reproductive traits from treatments evolving in high and low density, this study showcases the effect of conspecific interactions on the evolution of sex, since pre- and post-copulatory sexual selection are expected to be higher at higher densities. They measured mating frequency, latency to copulation, female longevity, and female lifetime reproductive success in both withinpopulation matings and between-population matings from the same density treatment. They found that female longevity and lifetime reproductive success decreased with increasing 
population density, but that the effect was stronger in between-population compared to withinpopulation crosses, suggesting that sexual conflict contributes to the evolution of reproductive isolation. In addition, mating frequency was higher and latency to copulation and female mating rejection was lower in crosses within populations, compared to crosses between populations, which suggests a preference for sympatric males contrast, populations maintained in monogamy did not differ in any trait, when comparing pairings within and between populations.

In this case it is impossible to disentangle the effects of density per se from the effects of an increased number of reproductive interactions, which means that we cannot identify the exact ecological driver of evolution involved in the eco-evolutionary feedback. However, density and number of matings should often be confounded in nature, which means that this example is still instructive, and demonstrates that interactions between conspecifics may mediate ecoevolutionary feedbacks: eco (high density) to evo (increased sexual conflict and sexually antagonistic coevolution) to eco (population differentiation and potential for local adaptation).

\section{Conclusions}

Researchers are often biased in terms of framing experiments within a single context (e.g., how ecological factors affect the strength or mode of sexual selection), rather than trying to reverse the direction of causality (e.g. how sexual selection can change the ecological context) or looking for feedback loops. Although we have here presented several examples where eco-evolutionary feedbacks of sexual selection have been unraveled, we also feel that the field is far from exploiting all its potential. To do so, there are some challenges ahead.

As highlighted previously, especially (but not exclusively) in the context of conspecific interactions it is difficult to decide what should constitute an "ecological" versus an "evolutionary" trait. In many cases, it is assumed that ecological processes operate at short 
timescales and evolutionary processes at long timescales. However, the examples discussed above highlight the fact that this is a false dichotomy in many cases, and that the same trait may even be considered both "ecological" and "evolutionary", depending on the context. In addition, the continuous nature of the eco-evolutionary dynamics means that the choice of any particular starting point for a feedback loop is essentially arbitrary. For example, in the previous section, we discussed an example where predation pressure leads to increased male mating harassment in guppies, causing a change in female diet as a result of avoiding males. However, one could just as easily start with male harassment as an ecological trait driving an evolutionary change in female avoidance behaviour, which then selects for increased sneaky mating behaviour in males (female avoidance is a driver of selection for male mating behaviour; So, the first scenario focuses on males being initially evolutionary objects (of predation) and then ecological drivers (of female behaviour), while the second scenario focuses on males being first an ecological driver of female evolution and then objects of evolved females. One might then wonder what the advantage is of defining a trait as an "ecological" or "evolutionary" driver/object in the first place. We would argue that some pragmatism is necessary here, to provide a compromise between biological relevance and conceptual precision. Developing a conceptual model of how a loop is thought to operate, and what factors are acting as drivers or objects at a given point, is valuable in terms of setting up explicit hypotheses and designing experiments but may not be relevant biologically. Once a particular causal relationship has been established from data, then the next stage in the loop can be considered, acknowledging but then setting aside the already established causal relationship, and the role of a particular factor as a driver or object may change. In our example, the existence of predation as an ecological trait leads to different 
outcomes in the male-female conflict. In this case, one can argue that it is a biologically sound point to start.

In addition, although eco-evolutionary feedbacks of sexual selection are probably common, it is difficult to address the whole feedback loop in a single study. Enough must be known about the system to identify potential drivers and their objects in order to design appropriate experimental manipulations and decide what phenotypes to track. Once a response to an ecological change has been measured, additional manipulations will often be necessary to determine the chain of causality. Finally, to confirm that the changes that have been observed are truly evolutionary in nature, the existence of a genetic response must be documented. Negative frequencydependence of alternative reproductive tactics are particularly tractable for understanding feedback loops, since their dynamics are often rapid and changes in allele frequencies can be measured using morph frequencies as a proxy. For example, in the Blue-tailed damselfly Ischnura elegans, male mating harassment mediates the frequency of the three female morphs, one of which (Androchrome) is a male mimic and the other two are more cryptic (Infuscans and Infuscans-obsoleta, collectively known as Gynochromes). When Gynochromes are at high frequency, males develop a search image for them, and they experience increased male mating harassment. This leads to decreased fecundity in Gynochromes, reducing their frequency in the next generation. Once Androchrome frequency is sufficiently high, males develop a search image for them instead, resulting in increased harassment and decreased fecundity against these females, and so on. Recognition of male-mimic Androchromes as female is additionally influenced by their frequency relative to the male model, and so population density and sex ratio also influence feedbacks of sexual selection in this system. Understanding their dynamics has required a combination of extensive field work and experimental manipulations in order to establish that: frequency changes in the field are consistent with negative-frequency dependence males develop a search image according to exposure to the female morphs female 
harassment levels differ according to their frequency and the population density and increased mating rate reduces female fecundity In this example, one "turn" of the feedback loop is constituted by a switch in male mate preference (an ecological change, from the point of view of females), causing a decrease in fecundity in the preferred high-frequency morph and subsequent decline in frequency of that morph (evolutionary response), until the switching point is reached and male preference switches back (ecological change as a result of the evolutionary response). This sort of feedback loop can be used as a conceptual guide to other types of feedback loops, in that one must first determine what factor is acting as the initial selective driver, determine what trait(s) are the object of this selection, demonstrate a causal evolutionary response in the object as a result of selection, and then determine how the altered trait(s) themselves constitute a new selective driver for trait(s) in another set of actors (i.e. the opposite sex, other species in the community).

\section{Future perspectives}

Sexual selection appears to play a dual role in evolutionary biology, having been shown to have the potential to both enhance and constrain adaptation (e.g. speciation (e.g. and extinction (e.g. Our understanding of when sexual selection will have an enhancing or constraining effect is currently incomplete and may be partly resolved by more in-depth study of feedback loops of sexual selection. To conclude, we highlight some possibly unexpected effects of sexual selection within evolutionary ecology, in order to illustrate how thinking about feedback loops may be valuable in moving the field forward and inspire others to do the same.

Sexual selection is usually thought to enhance adaptation via more efficient purging of deleterious mutations in males However, adaptation to a new sexually selected trait could also lead to exploitation of a new ecological niche. For example, the evolution of a preference for a 
novel ornament could result in a sensory bias towards food resources of a similar colour, allowing the evolution of novel diet choices and subsequent specialization. This appears to have occurred in Goodeidae fish, where there is evidence that males have evolved an ornament which resembles a damselfly larvae, resulting in sensory exploitation of females Females in species with more conspicuous ornaments have evolved resistance to this trait, and shifted their food preference away from damselfly larvae Another, perhaps more common, scenario could be when there is sexual selection for increased body size, which then leads to the ability to exploit larger food sources and therefore an altered niche. Similarly, Therry et al. tudied geographic variation in morphological characteristics and flight performance, as well as sexual selection on these traits in males of the damselfly Coenagrion scitulum. They found evidence of consistent sexual selection for longer flight duration, which is probably an effect of scramble competition in this species. Since this species is also currently expanding its range northwards, it is likely that sexual selection contributes to range expansion via this selection for increased flight performance.

A recent meta-analysis as shown that the intensity of sexual selection is related to species richness, but the exact mechanism by which sexual selection promotes speciation is unclear. A classic model by Lande suggests that populations can diverge in allopatry via the evolution of different sexual traits, while Turner and Burrows odelled divergence in sympatry via a switch in female mate preference and subsequent divergent selection on the male sexual trait. Other models suggest that sexual selection could be important in sympatric speciation by promoting assortative mating by habitat However, the feedback loops discussed above suggest other possible routes whereby sexual selection could affect the evolution of reproductive isolation. For example, Fisherian runaway effects could allow the evolution of reproductive isolation and subsequent specialization between populations of generalists. In closely related populations living in different environments, migration and subsequent genetic homogenization can prevent 
the evolution of specialization on different resources, causing the populations to remain as a single generalist species In this sort of situation, Fisherian runaway could lead to the evolution of different ornaments in different populations, favouring assortative mating even in the face of low levels of migration. Once some degree of assortative mating has evolved, genetic homogenization is reduced, and the populations are free to adapt to the most abundant resource in their respective environment, resulting in ecological specialization. In this case, the evolution of divergence in sexual traits precedes ecological specialization rather than following from it $r$ being independent of it

Finally, sexual selection and particularly sexual conflict have the potential to influence extinction rates via eco-evolutionary feedbacks. Under sexual conflict, males may harm females and decrease their fecundity (e.g. A situation similar to predator-prey dynamics can then arise via density-dependent interactions. For example, in a high-density population, male mating harassment may reduce female fertility, leading to a decrease in overall population size. As density decreases, food resources will become more abundant, causing a later increase in population size, and leading to increased male mating harassment again. This type of increased demographic instability as a result of sexual interactions has been predicted to increase the extinction rate in small populations despite possible benefits of sexual selection via an increased rate of adaptation.

In sum, evolutionary biologists have been aware that sexual selection may affect natural selection ever since Darwin, and that ecological adaptations will therefore influence the course and nature of sexual selection. However, as discussed above, there are also many hints in the literature that sexual selection and sexual conflict can in their turn influence the ecology of populations and species. The balance between sexual selection and sexual conflict is likely an important factor determining if eco-evolutionary feedbacks of sexual selection have positive or negative effects on populations (e.g. Yun et al. 2018). It is also conceivable that the nature of 
the initial driver of evolutionary change (abiotic, heterospecific, or conspecific) has an impact on whether effects of sexual selection are generally positive or negative, but this bears further investigation. Developing a conceptual framework for identifying and characterizing ecoevolutionary feedbacks of sexual selection will hopefully help researchers in evolutionary ecology to think carefully about the effects that sexual selection may have in their specific study system, and study more systematically how sexual selection can contribute to various evolutionary processes such as ecological adaptation, speciation, and extinction.

\section{References}

Bush, G. L. 1994. Sympatric speciation in animals: new wine in old bottles. - Trends Ecol. Evol. 9: 285-288.

Chenoweth, S. F. et al. 2015. Genomic evidence that sexual selection impedes adaptation to a novel environment. - Curr. Biol. 25: 1860-1866.

Darwin, C. 1871. The descent of man and selection in relation to sex. - D. Appleton and Company.

Dussault, G. V. and Kramer, D. L. 1981. Food and feeding behavior of the guppy, Poecilia reticulata (Pisces: Poeciliidae). - Can. J. Zool. 59: 684-701.

Fussmann, G. F. et al. 2007. Eco-evolutionary dynamics of communities and ecosystems. Funct. Ecol. 21: 465-477.

Garcia, C. M. and Lemus, Y. S. 2012. Foraging costs drive female resistance to a sensory trap. - 
Proc. R. Soc. B 279: 2261-2268.

Gosden, T. P. and Svensson, E. I. 2007. Female Sexual Polymorphism and Fecundity Consequences of Male Mating Harassment in the Wild. - PLoS One 2: e580.

Gosden, T. P. and Svensson, E. I. 2009. Density-Dependent Male Mating Harassment, Female Resistance, and Male Mimicry. - Am. Nat. 173: 709-721.

Grieshop, K. et al. 2016. Strong sexual selection in males against a mutation load that reduces offspring production in seed beetles. - J. Evol. Biol. 29: 1201-1210.

Heathcote, R. J. P. et al. 2016. Male behaviour drives assortative reproduction during the initial stage of secondary contact. - J. Evol. Biol. 29: 1003-1015.

Hendry, A. P. 2016. Eco-Evolutionary Dynamics. - Princeton University Press.

Hereford, J. 2009. A Quantitative survey of local adaptation and fitness trade-offs. - Am. Nat. 173: 579-588.

Janicke, T. et al. 2018. Sexual selection predicts species richness across the animal kingdom. Proc. R. Soc. B 285: 20180173.

Kirkpatrick, M. and Nuismer, S. L. 2004. Sexual selection can constrain sympatric speciation. Proc. R. Soc. B 271: 687-93.

Lande, R. 1981. Models of speciation by sexual selection on polygenic traits. - Proc. Natl. Acad. Sci. U. S. A. 78: 3721-5.

Le Rouzic, A. et al. 2015. Evolutionary time-series analysis reveals the signature of frequencydependent selection on a female mating polymorphism. - Am. Nat. 185: E182-96.

Lesna, I. and Sabelis, M. W. 1999. Diet-dependent female choice for males with 'good genes' in 
a soil predatory mite. - Nature 401: 581-584.

Lion, S. 2017. Theoretical approaches in evolutionary ecology: environmental feedback as a unifying perspective. - Am. Nat. 191: 21-44.

Lumley, A. J. et al. 2015. Sexual selection protects against extinction. - Nature 522: 470-473.

Maan, M. E. et al. 2010. Female mating preferences and male coloration covary with water transparency in a Lake Victoria cichlid fish. - Biol. J. Linn. Soc. 99: 398-406.

Magurran, A. E. and Seghers, B. H. 1994. Sexual Conflict as a Consequence of Ecology: Evidence from Guppy, Poecilia reticulata, Populations in Trinidad. - Proc. R. Soc. B 255: 31-36.

Martin, O. Y. and Hosken, D. J. 2003. The evolution of reproductive isolation through sexual conflict. - Nature 423: 979-982.

Martin, O. Y. and Hosken, D. J. 2004. Reproductive consequences of population divergence through sexual conflict. - Curr. Biol. 14: 906-10.

Martínez-Ruiz, C. and Knell, R. J. 2017. Sexual selection can both increase and decrease extinction probability: reconciling demographic and evolutionary factors. - J. Anim. Ecol. 86: 117-127.

Palkovacs, E. P. and Hendry, A. P. 2010. Eco-evolutionary dynamics: intertwining ecological and evolutionary processes in contemporary time. - F1000 Biol. Rep. 2: 1.

Pelletier, F. et al. 2009. Eco-evolutionary dynamics. - Philos. Trans. R. Soc. B Biol. Sci. 364: 1483-9.

Pitnick, S. and García-González, F. 2002. Harm to females increases with male body size in Drosophila melanogaster. - Proc. R. Soc. B 269: 1821-8.

Plesnar-Bielak, A. et al. 2012. Mating system affects population performance and extinction 
risk under environmental challenge. - Proc. R. Soc. B 279: 4661-7.

Pontarp, M. et al. 2015. The biogeography of adaptive radiations and the geographic overlap of sister species. - Am. Nat. 186: 565-581.

Rice, W. R. 1996. Sexually antagonistic male adaptation triggered by experimental arrest of female evolution. - Nature 381: 232-234.

Rodrigues, L. R. et al. 2016. Integrating Competition for Food, Hosts, or Mates via Experimental Evolution. - Trends Ecol. Evol. 31: 158-170.

Seehausen, O. et al. 2008. Speciation reversal and biodiversity dynamics with hybridization in changing environments. - Mol. Ecol. 17: 30-44.

Seghers, B. H. 1974. Geographic variation in the responses of guppies (Poecilia reticulata) to aerial predators. - Oecologia 14: 93-98.

Selz, O. M. et al. 2014. Female preference for male color is necessary and sufficient for assortative mating in 2 cichlid sister species. - Behav. Ecol. 25: 612-626.

Slobodkin, L. B. 1961. Growth and Regulation of Animal Populations. - Holt, Rinehart and Winston.

Svensson, E. I. et al. 2005. Female polymorphism, frequency dependence, and rapid evolutionary dynamics in natural populations. - Am. Nat. 165: 567-76.

Takahashi, Y. et al. 2014. Evolution of increased phenotypic diversity enhances population performance by reducing sexual harassment in damselflies. - Nat. Commun. 5: 4468.

Therry, L. et al. 2014. Integrating large-scale geographic patterns in flight morphology, flight characteristics and sexual selection in a range-expanding damselfly. - Ecography 37: 
1012-1021.

Turner, G. F. and Burrows, M. T. 1995. A Model of Sympatric Speciation by Sexual Selection. Proc. R. Soc. B 260: 287-292.

van Gossum, H. et al. 2001. Reversible frequency-dependent switches in male mate choice. Proc. R. Soc. B 268: 83-5.

Yun, L. et al. 2018. Competition for mates and the improvement of nonsexual fitness. - Proc. 
Natl. Acad. Sci. U. S. A. 115: 6762-6767. 
Figure 1. Different traits, abiotic or biotic, can function as the driver or the object of an ecoevolutionary process. Identifying the driver and object of a process can be easy when one of the traits is abiotic (a), but it can be hard when several traits are biotic (b and c). a) An abiotic factor drives the evolution of a trait (eco-evo process). b) and c) A biotic factor (one sex/one species), drives the evolution of another biotic factor (the other sex/the other species). Each biotic factor can be the driver or the object of selection simultaneously (1), or sequentially (2). If 1 , two traits jointly evolve in response to each other (eco-to-evo process). If 2 , there is an eco-evolutionary feedback: A first actor (males, in the example) drives the evolution of a first object of selection (females, in the example) (eco-to-evo); In turn, the evolved object of selection will become the new ecological driver for the first actor (evo-to-eco), causing the first actor's (now the object of 
selection) evolution (eco-to-evo). Distinct colour tones represent different genotypes; distinct colours represent different species; dashed lines circle ecological drivers; full lines circle objects of selection; dashed arrows point from ecological drivers to objects of selection; grey arrows 
represent evolutionary change; bars graphs indicate the fitness distribution of genotypes.

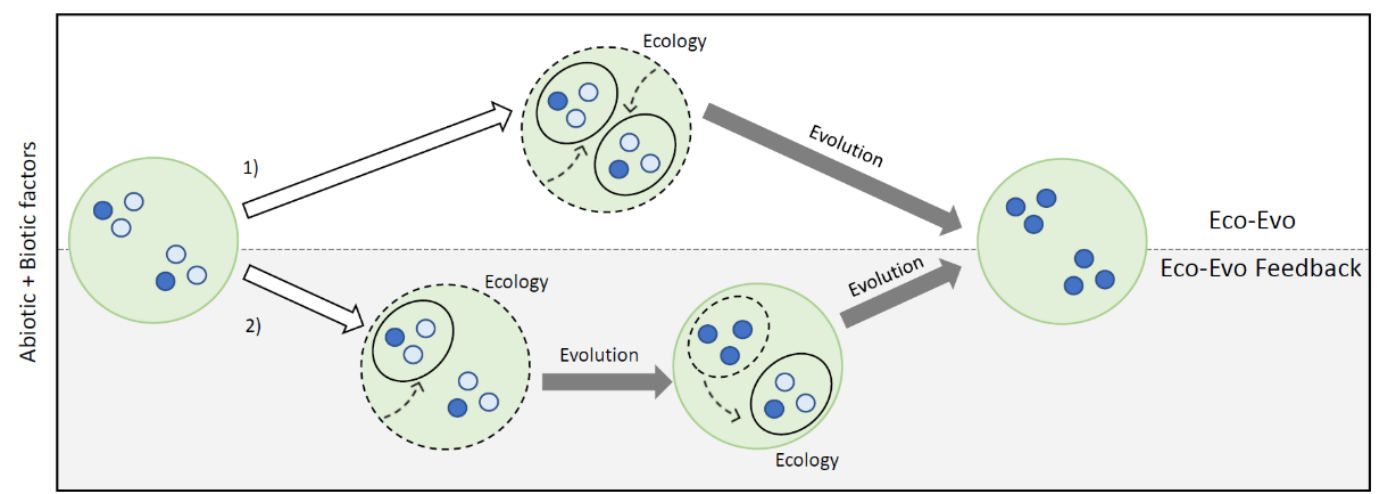


Figure 2. The same evolutionary outcome can result from different eco-evolutionary processes. In this example, a population in a specific initial state can evolve a particular evolutionary outcome via two paths, 1) or 2). Indeed, a change in two traits can be due to 1) a common selective pressure (in here abiotic) or 2) a first trait that is selected, and another that responds to the change in this selected trait. If 1 ), there is an eco-evolutionary process occurring, and no feedback was reached. If 2), the responding trait is a correlated response to selection on the selected one, and this is a good example of an eco-evolutionary feedback. Distinct colour tones represent different genotypes. dashed lines circle ecological drivers; full lines circle objects of selection; dashed arrows point from ecological drivers to objects of selection; grey arrows represent evolutionary change; white arrows indicate alternative processes. 